Rev. Bras. Saúde Prod. Anim., Salvador, v.15, n.3, p.689-695 jul./set., 2014 http://www.rbspa.ufba.br ISSN 15199940

\title{
Ocorrência de hematomas em carcaças de bovinos transportados por duas distâncias
}

\section{Occurrence of carcasses bruises of beef cattle transported by two distances}

\author{
MOREIRA, Paulo Sérgio Andrade ${ }^{1 *}$; POLIZEL NETO, Angelo ${ }^{1}$; MARTINS, Lilian \\ Rigatto $^{1}$; LOURENÇO, Fabio José ${ }^{2}$; PALHARI, Celina ${ }^{3}$; FARIA, Felipe Ferreira ${ }^{3}$
}

\author{
${ }^{1}$ Universidade Federal de Mato Grosso, Instituto Ciências da Saúde, docente do Programa Pós-Graduação \\ em Zootecnia, Campus Sinop,- Mato Grosso. Brasil \\ ${ }^{2}$ Universidade Federal de Mato Grosso, Instituto Ciências da Saúde, Curso Medicina Veterinária, Campus \\ Sinop, Mato Grosso. Brasil \\ ${ }^{3}$ Universidade Federal de Mato Grosso, Instituto Ciências da Saúde, discente do Programa Pós-Graduação \\ em Zootecnia, Campus Sinop, Mato Grosso. Brasil \\ *Endereço para correspondência: paulomoreira@ufmt.br
}

RESUMO

O Objetivo foi determinar a ocorrência de hematomas em carcaças de bovinos transportados por duas distâncias e abatidos no norte do estado do Mato Grosso. Foram avaliados 624 bovinos machos de 30 a 36 meses oriundos de distâncias variadas até o frigorífico, sendo 352 animais oriundos de distâncias menores que $200 \mathrm{~km}$ e 272 animais transportados por mais de $200 \mathrm{~km}$. Para avaliação dos hematomas nas carcaças, seguiuse o critério descrito pela AUS-MEAT (2005), sendo as lesões classificadas de acordo com a sua localização na carcaça (traseiro, dianteiro ou costado). A relação de hematomas por grupo aumentou com a maior distância de transporte, sendo $43,75 \%$ e $95.58 \%$ de animais com hematomas para distâncias menores e maiores que $200 \mathrm{~km}$, respectivamente. Quando analisada a região do hematoma, verificou-se 7,6 e 17,6 observações na região do dianteiro $(\mathrm{P}=0,035)$ e 30,6 e 101,0 observações na região do traseiro $(\mathrm{P}=0,009)$ para distâncias menores e maiores que $200 \mathrm{~km}$, respectivamente. Portanto, a distância do transporte de bovinos até o abate teve influência sobre a quantidade de hematomas apresentados, principalmente na região do traseiro.

Palavras-chave: abate, bem-estar, contusão, qualidade, transporte

\section{SUMMARY}

This study aimed to determine the occurrence of beef cattle carcasses bruises on transported by two distances and slaughtered in northern Mato Grosso State. In total, 624 male animals were evaluated 30 to 36 months come from varying distances to slaughterhouse, with 352 animals originating from within $200 \mathrm{~km}$ and 272 animals above $200 \mathrm{~km}$. To review the injuries on carcasses, followed the criteria described by AUS -MEAT (2005), the lesions were classified according to their location in the carcass (forequarter, spare ribs and hindquarter). The relationship of lesions per group increased with greater distance of transport, being $43.75 \%$ and $95.58 \%$ of animals with carcass bruised, respectively, to less and more than $200 \mathrm{~km}$ of trip. When analyzed the region of the hematoma, observed 7.6 e 17.6 carcass bruises in the forequarter regions $(\mathrm{P}=0.035)$ and $30,6 \mathrm{e}$ 101,0 in the hindquarter regions $(\mathrm{P}=0.009)$ to near and longer distance, respectively. It is concluded that the transport distance to slaughter increases the number of carcass bruises, mainly, in the hindquarter.

Keywords: slaughter, truck, meat, contusion, quality. 


\section{INTRODUÇÃO}

A qualidade visual e a gustativa são influenciadas por fatores ante mortem, ou intrínsecos, vinculados ao genótipo dos animais e ao ambiente em que se desenvolvem; e os post mortem, ou extrínsecos, que são os procedimentos técnicos adotados pelos matadourosfrigoríficos, até o consumidor final (FELÍCIO, 1997). Hoje em dia, muita ênfase tem sido dada para as consequências econômicas do manejo e transporte deficiente dos animais. (GALLO, 2008)

Segundo Roça (2001), o transporte rodoviário, em condições desfavoráveis, pode provocar contusões nos animais, morte, perda de peso e estresse. As altas temperaturas, maiores distâncias de transporte e diminuição do espaço ocupado por animal também contribuem para problemas no transporte e aumento das contusões. A distância pode causar estresse psicológico quando os animais são submetidos a um novo ambiente e a um novo manejo, e, também, estresse físico, machucados, temperaturas indesejáveis e restrição alimentar. (GRANDIN, 2006)

A distância percorrida pelos animais entre a fazenda e o local de abate pode exercer influência sobre a perda de peso, além de elevar a ocorrência de contusões em várias regiões, e de variados diâmetros, afetando a qualidade da carne. (HUERTAS et al., 2010) Para Andrade et. al. (2008), além de considerar a distância como importante fator nas causas de contusões é importante considerar o movimento dos bovinos dentro do caminhão durante a aceleração e desaceleração. Essas carcaças com fraturas e/ou hematomas resultarão em perdas financeiras, não apenas para o frigorífico, mas também para os pecuaristas. (ANDRADE et al. 2008; STRAPPINI et al., 2010) Um aspecto prático e objetivo utilizado para avaliar as condições do pré-abate é a quantificação das contusões observadas nas carcaças dos animais abatidos. A presença de hematomas é um indicativo de manejo inadequado, o qual pode ter ocorrido em qualquer etapa do processo pré-abate, além disso, a carne da região atingida pela contusão tem uma aparência feia e desagradável não sendo apta para o consumo humano e, durante a inspeção post mortem, é submetida a um toalete intenso, podendo até ser desclassificada com prejuízo financeiro. (STRAPPINI et al., 2009)

O objetivo foi determinar a ocorrência de hematomas e sua localização em carcaças de bovinos transportados por duas diferentes distâncias como um indicativo do manejo pré-abate no norte do estado do Mato Grosso.

\section{MATERIAL E MÉTODOS}

O trabalho foi realizado no Frigorífico Redentor localizado às margens da Rodovia BR163, na cidade de Guarantã do Norte, estado do Mato Grosso, entre os meses de maio a julho de 2013. Os lotes avaliados eram provenientes das cidades localizadas no estado do Mato Grosso: Guarantã do Norte, Nova Guarita, Terra Nova, Matupá, Peixoto de Azevedo, Alta Floresta; e das cidades de Altamira e Novo Progresso, localizadas no estado do Pará.

Foram utilizados 624 bovinos machos castrados da raça Nelore, de 30 a 36 meses de idade, divididos em dois grupos de acordo a distância percorrida. Grupo de animais $(n=352)$ transportados por distâncias inferiores a $200 \mathrm{~km}$ e o outro grupo de animais $(n=272)$ oriundos de distâncias superiores a $200 \mathrm{~km}$.

No momento da chegada dos caminhões, os animais foram descarregados e pesados em balança 
individual, marcados com a numeração específica para facilitar a identificação do local de origem. Os hematomas foram avaliados devido ao tempo de ocorrências dos mesmos, sendo somente contabilizados os hematomas recentes, ou seja, aqueles que apresentavam colorações vermelhas ou arroxeados, caracterizando erros no embarque ou transporte.

Foram considerados hematomas as lesões traumáticas com ruptura de vasos sanguíneos, com acúmulo de sangue e soro, sem descontinuidade cutânea. (NANNI COSTA et al., 2006)

A detecção dos hematomas foi realizada nas linhas de rotina de inspeção de carcaças observando as faces medial e lateral da parte caudal da meia carcaça e as faces medial e lateral da parte cranial da meia carcaça. Após a inspeção das meias carcaças, estas foram encaminhadas para a plataforma de toalate, onde os hematomas, quando necessários, foram retirados à faca.

As lesões presentes na carcaça eram identificadas visualmente de acordo com a localização na região da carcaça (dianteiro, costado ou traseiro), tendo como base um padrão fotográfico e critérios descritos por AUS-MEAT (2005), sendo determinada a idade das lesões que apresentavam coloração avermelhada ou arroxeada como recente e coloração amarelada de antiga.

Os dados de peso vivo, rendimento de carcaça e número de hematomas no dianteiro, costado e traseiro em função da distância percorrida foram submetidas à Análise de Variância (ANOVA) ao nível de 5\% de significância, utilizando-se o Software R (R DEVELOPMENT CORE TEAM, 2010), utilizando o seguinte modelo estatístico:

$y_{i j}=\mu+D_{i}+e_{i j}$, em que:

$\mathrm{y}_{\mathrm{ij}}=$ observação da amostra $i j$;

$\mu=$ média geral;

$\mathrm{D}_{\mathrm{i}}=$ distância percorrida $(i=1(<200 \mathrm{~km})$; $2(>200 \mathrm{~km})$;

eij = erro associado à observação ij.

\section{RESULTADOS E DISCUSSÃO}

A Tabela 1 demonstra os municípios de origem dos grupos observados, sua distância em relação ao Frigorífico, número total de animais por lote, a quantidade de hematomas observados, o número de animais com hematomas e a relação em porcentagem de animais com hematomas por lote. Quando se avalia a quantidade de hematomas nos animais provenientes de distâncias superiores a $200 \mathrm{~km}$, os números são superiores aos que percorreram distâncias menores. Há uma diferença $(\mathrm{P}<0,05)$ quando analisamos a relação de número de hematomas nos animais transportados a distância inferior a $200 \mathrm{~km}$, demonstrando que a relação de hematomas com o número de animais sofreu influência da distância de transporte, sendo $43,75 \%$ e $95,58 \%$ lesões por animal abatido, respectivamente para os animais inferior e superior a $200 \mathrm{~km}$ de transporte.

Estes resultados estão relacionados com fatores psicológicos e físicos como a movimentação transporte, o manejo e a distância que promovem o estresse e predispõem os animais a sofrer lesões. (MUCHENJE et al., 2009; NDOU et al., 2011)

Discordando desse resultado, Romero et al.2012, avaliando a presença de lesões em bovinos transportados no Chile por 9 a 41h, concluíram que o tempo de transporte não influenciou a quantidade de lesões nos animais, verificaram que características como a densidade de carga durante o transporte, a estadia na planta e o sexo são possíveis fatores de risco que influenciaram sobre a presença de contusões sobre os animais estudados.

Neste mesmo sentido Almeida et. al. (2008), não encontraram diferença entre a distância percorrida com a presença das lesões detectada, predominando as lesões recentes, indicando que os 
eventos que causaram a lesão ocorreram em curto espaço de tempo em relação ao abate do animal, incluindo desde o manejo pré-embarque até o momento do abate em si.

Bertoloni et al., (2012), ao avaliar o índice de hematomas das carcaças, observaram que todos os tratamentos estudados proporcionaram elevada incidência, visto que todos apresentarem taxa superior a $60 \%$, com destaque para carretas double deck, na qual nas distâncias curtas (até $130 \mathrm{~km}$ ) e longas (de 180 a $250 \mathrm{~km}$ ) apresentaram, respectivamente, 100 e $91,35 \%$, o que demonstra redução do bem-estar e qualidade de carcaça.

Tabela 1. Relação das cidades de origem, distâncias percorridas, número de animais e número de hematomas observados e relação número com hematomas no lote

\begin{tabular}{lcccccc}
\hline \multicolumn{1}{c}{ Município } & $\begin{array}{c}\text { Distância } \\
(\mathrm{km})\end{array}$ & $\begin{array}{c}\text { Total de } \\
\text { animais }\end{array}$ & $\begin{array}{c}\mathrm{N}^{\circ} \text { de } \\
\text { hematomas }\end{array}$ & $\begin{array}{c}\text { Animais com } \\
\text { hematomas }\end{array}$ & $\begin{array}{c}\% \\
\text { Grupo }\end{array}$ & P \\
\hline Guarantã do Norte - MT & 54 & 34 & 28 & 22 & - & - \\
Peixoto de Azevedo - MT & 78 & 102 & 24 & 06 & - & - \\
Matupá - MT & 90 & 85 & 54 & 03 & - & - \\
Terra Nova do Norte - MT & 122 & 34 & 34 & 30 & - & - \\
Nova Guarita - MT & 141 & 57 & 78 & 55 & - & - \\
Alta Floresta - MT & 171 & 40 & 51 & 38 & - & - \\
Total <200km & & 352 & 269 & 154 & 43,75 & 0,028 \\
\hline Novo Progresso - PA & 322 & 119 & 134 & 115 & - & - \\
Altamira - PA & 1100 & 153 & 189 & 145 & - & - \\
Total > 200km & - & 272 & 323 & 260 & 95,58 \\
\hline
\end{tabular}

Andrade e Coelho, 2011, avaliando bovinos oriundos de diferentes distâncias encontraram que com menos de $100 \mathrm{~km}$ do matadouro-frigorífico foram analisados 2724 carcaças e 2499 tiveram contusões demonstrando um percentual de $91,74 \%$. Nos municípios de 100 a $200 \mathrm{~km}$ de distância do matadouro-frigorífico foram analisados 738 carcaças e 691 tiveram contusões demonstrando um percentual de $93,63 \%$.

Nos municípios com 200 a 300km de distância do matadouro-frigorífico foram analisados 23 carcaças e 21 tiveram contusões, demonstrando um percentual de 91,3\%. Portanto, em média, 92,1\% dos animais avaliados apresentaram lesões, independente da distância de transporte percorrida, sendo esse valor similar ao apresentado neste trabalho com animais que foram transportados em distâncias superiores a $200 \mathrm{~km}$.

Os dados apresentados na Tabela 2 demonstram que os animais oriundos de diferentes distâncias de transporte apresentavam peso vivo médio semelhante no momento do abate, $504,1 \mathrm{~kg}$ e $516,7 \mathrm{~kg}$, respectivamente, para os animais $<200 \mathrm{~km}$ e $>200 \mathrm{~km}$. Essa mesma semelhança é verificada no rendimento de carcaça (RC) entre os grupos avaliados, sendo $55,7 \%$ e $57,7 \%$. $\mathrm{O}$ rendimento de carcaça está sujeito à grande variação, por influência de diversos fatores, de forma que valores diferentes serão obtidos, se o rendimento for calculado em relação ao peso vivo ou ao peso de corpo vazio (livre da digesta).

Contudo, ao analisarmos o peso de carcaça quente (PCQ), os animais transportados por distâncias maiores $(298,0 \mathrm{~kg})$ tiveram diferença $(\mathrm{P}=0.038)$ 
em relação aos animais oriundos de distância inferior a $200 \mathrm{~km}(279,98 \mathrm{~kg})$, sendo esses animais com maior índice de gordura e apresentaram uma quebra menor em relação ao peso vivo pré abate. Isso pode ser explicado devido ao tempo de permanência nos caminhões da fazenda até o frigorífico, pois os valores com base no peso vivo são afetados pelo tempo de jejum e tipo de dieta. Preston e Willis (1982) mostraram que o rendimento de carcaça aumenta com o peso de abate e o nível de engorda. Os frigoríficos remuneram o produtor pelo PCQ sendo um parâmetro importante para o pecuarista, sendo PCF um parâmetro da indústria para avaliar o rendimento de desossa e cortes cárneos.

Tabela 2. Peso vivo médio de abate $(\mathrm{kg})$, peso de carcaça quente $(\mathrm{kg})$, rendimento de carcaça $(\%)$ e número de hematomas conforme região em bovinos jovens abatidos com transporte inferior e superior a $200 \mathrm{~km}$

\begin{tabular}{lccc}
\hline \multicolumn{2}{c}{ Grupo } & \\
\hline \multirow{2}{*}{ Item } & Até $200 \mathrm{~km}$ & $>200 \mathrm{~km}$ & $\mathrm{P}$ \\
\hline Peso vivo médio & $\mathrm{X} \pm \mathrm{SD}$ & $\mathrm{X} \pm \mathrm{SD}$ & 0,714 \\
PCQ $(\mathrm{Kg})$ & $504,15 \pm 32,02$ & $516,74 \pm 34,77$ & 0,038 \\
RC $(\%)$ & $279,98 \pm 9,38$ & $298,05 \pm 9,44$ & 0,335 \\
Hemat. dianteiro & $55,71 \pm 3,9$ & $17,76 \pm 2,8$ & 0,035 \\
Hemat. costado & $7,6 \pm 5,9$ & $6,0 \pm 11,0$ & 0,835 \\
Hemat. traseiro & $5,4 \pm 2,9$ & $101,0 \pm 61,8$ & 0,009 \\
\hline P $<0,05>$ & $30,6 \pm 13,9$ & &
\end{tabular}

$\mathrm{P}<0,05>$.

Quanto ao número de contusões observado nos animais, houve diferença entre os grupos, analisando a região onde foram verificadas a contusão, as regiões do dianteiro $(\mathrm{P}=0,035)$ e traseiro $(\mathrm{P}=0,009)$ também tiveram diferença, sendo que, os animais com maior distância de transporte também apresentaram o maior número de hematomas, na região do dianteiro 17,6 e traseiro 101,0; enquanto que os animais transportados em menor distância 7,6 e 30,6 , respectivamente.

Concordando com estes resultados, Romero et al. (2012), trabalhando com prevalência de lesões e bem-estar préabate encontraram que, em relação à localização, a perna e o lombo (traseiro) foram as áreas mais atingidas (70,2\%), áreas de corte de maior valor comercial, enquanto que Minka \& Ayo (2007), trabalhando com animais mestiços Bos taurus indicus, encontraram valores inferiores aos encontrados no presente trabalho na região do traseiro $12,5 \%$ para o Chile e 20,3\% para África Ocidental. Nesse mesmo sentido, Bertoloni et al. (2012), avaliando carcaças de 120 bovinos, encontraram maiores número de hematomas na região do traseiro, com animais submetidos a diferentes distâncias de transporte.

As observações na região do costado não apresentaram diferença $(\mathrm{P}=0,835)$ entre os grupos analisados. Segundo Gregory, 2008, a maioria das contusões encontradas na carcaça pode ser prevenida quando implementados programas de boas práticas de manejo, como descorna dos animais, manutenção preventiva de equipamentos e instalações, infraestrutura de transporte especializada, diminuição de tempo de transporte e permanência na planta frigorífica. Colaborando com essa afirmação, Ferguson \& Warner (2008) concluíram 
Rev. Bras. Saúde Prod. Anim., Salvador, v.15, n.3, p.689-695 jul./set., 2014 http://www.rbspa.ufba.br ISSN 15199940

que $50 \%$ das contusões sofridas na carcaça dos bovinos ocorreram após a entrada dos animais na planta do frigorífico.

A distância do transporte de bovinos até o abate teve influência sobre a quantidade de hematomas apresentados, principalmente na região do traseiro, portanto, medidas de melhoria no bemestar dos animais no manejo pré-abate é fundamental para produção de uma carcaça de qualidade.

\section{REFERÊNCIAS}

ALMEIDA, L.A.M.; PRATA, L.F.; FUKUDA, R.T.; VERARDINO, $\mathrm{H}$.

Manejo pré- abate de bovinos:

Monitoração de bem estar animal em frigoríficos exportadores - Perdas econômicas por contusões. Revista

Higiene Alimentar, v.22, n.164. 2008.

ANDRADE, E.N.; SILVA, R.A.M.S.; ROÇA, R.O.; SILVA, L.A. C.;

GONÇALVES, H.C.; PINHEIRO, R.S.B. Ocorrência de lesões em carcaças de bovinos de corte no Pantanal em função do transporte. Ciência Rural, v.38, n.7, p.1991-1996, 2008.

ANDRADE, J.; COELHO, H.E.; Ocorrência de Contusões Em Carcaças Bovinas E Suas Perdas Econômicas. Cadernos de Pós-Graduação da FAZU, p.332-338, 2011

AUS-MEAT. Handbook of Australian Meat. 7.ed. Sydney, 2005. 288p.

BERTOLONI, W.; SILVA, J.L.; ABREU, J.S.D.L. Bem-estar e taxa de hematomas de bovinos transportados em diferentes distâncias e modelos de carroceria no estado do Mato Grosso Brasil. Revista Brasileira de Saúde e Produção Animal, v.13, n.3, p.850-859. 2012.
FELICIO, P.E. Fatores ante e postmortem que influenciam na qualidade da carne vermelha. In: REUNIÃO ANUAL DA SOCIEDADE BRASILEIRA DE ZOOTECNIA, 30., 1997, Rio de Janeiro, RJ. Anais... Rio de Janeiro: Sociedade Brasileira de Zootecnia, 1997. p.43-52.

\section{FERGUSON D.M; WARNER R.D.}

Have we underestimated the impact of pre-slaughter on meat quality in ruminants. Meat Science, v.80. p.1219, 2008.

GALLO C. Transporte e bem-estar animal. Ciência Veterinária Tropical, v.11. p.70-79, 2008. Suppl. 1.

GRANDIN, T. Progress and challenges in animal handling and slaughter in the U.S. Applied Animal Behavior Science, v.100, p.129-139, 2006.

GREGORY, N. G. Animal welfare at markets and during transport and slaughter. Meat Science. v.80, p.2-11. 2008.

HUERTAS S.M.; GIL, A.D.; PIAGGIO, J.M. VAN EERDENBURG, F.J.C.M. Transportation of beef cattle to slaughterhouses and how this relates to animal welfare and carcase bruising in an extensive production system.

Animal Welfare, v.19, p.281-285, 2010.

MUCHENJE, V.; DZAMA, K.; CHIMONYO, M.; STRYDOM, P.E.; RAATS, J.G. Relationship between preslaughter stress responsiveness and beef quality in three cattle breeds. Meat Science, v.8, p.653-657, 2009.

MINKA, N.S.; AYO, JO. Effects of loading behaviour and road transport stress on traumatic injuries in cattle transported by road during the hot-dry season. Livestock Science, v.107, p.9195, 2007. 
Rev. Bras. Saúde Prod. Anim., Salvador, v.15, n.3, p.689-695 jul./set., 2014 http://www.rbspa.ufba.br ISSN 15199940

NANNI COSTA, L.L.O;; FIEGO, D.P.; TASSONE, F.; RUSSO, V. The relationship between carcass bruising in bulls and behavior observed during pre-slaughter phases. Veterinary Reserch Communications, v.30, p.379-381, 2006.

NDOU, S.P.; MUCHENJE, V.; CHIMONYO, M. Animal welfare in multipurpose cattle production systems and its implications on beef quality.

African Journal Biotechnology, v.10. p.1049-1064, 2011

PRESTON, T. R.; WILLIS, M.B. Intensive beef production. 2.ed. Oxford: Pergamon Press, 1982. 567p.

R DEVELOPMENT CORE TEAM. R:A Language and Environment for Statistical Computing. Vienna, Austria: R Foundation for Statistical Computing, 2010.

ROÇA, R.O. Abate humanitário de bovinos. Revista de Educação

Continuada do CRMV, v.4, n.2, p.7385, 2001.
ROMERO, M.H.; GUTIERREZ, C.; SANCHEZ, J.A. Evaluation of bruises as an animal welfare indicator during pre-slaughter of beef cattle. Revista Colombiana Ciencia Pecuária, v.24. p.93-101, 2012

STRAPPINI, A.C.; METZ, J.H.M. GALLO, C.B.; KEMP, B. Origin and assessment of bruises in beef cattle at slaughter. Animal, v.3, p 728-736, 2009.

STRAPPINI, A.C.; FRANKENA, K.; METZ, J.H.M.; GALLO, B; KEMP, B. Prevalence and risk factors for bruises in Chilean bovine carcasses. Meat Science, v.86, p.859-864, 2010.

Data de recebimento: 20/01/2014

Data de aprovação: 09/09/2014 